\title{
Influence of Resistance Training Variables on Excess Postexercise Oxygen Consumption: A Systematic Review
}

\author{
Paulo Farinatti, ${ }^{1,2}$ Antonio Gil Castinheiras Neto, ${ }^{1}$ and Nádia Lima da Silva ${ }^{2,3}$ \\ ${ }^{1}$ Graduate Program in Sciences of Physical Activity, Salgado de Oliveira University, \\ Rua Marechal 217, No. 2 Andar, 24030-060 Niterói, RJ, Brazil \\ ${ }^{2}$ Laboratory of Physical Activity and Health Promotion, Rio de Janeiro State University, \\ Rua São Francisco Xavier 524, Sala 8121F, 20550-900 Rio de Janeiro, RJ, Brazil \\ ${ }^{3}$ Faculty of Physical Education, Federal University of Juiz de Fora, Rua José Lourenço Kelmer, \\ s/n Campus Universitário, 36036-900 Juiz de Fora, MG, Brazil
}

Correspondence should be addressed to Paulo Farinatti; pfarinatti@gmail.com

Received 9 August 2012; Accepted 9 September 2012

Academic Editors: A. W. Midgley and A. A. Steiner

Copyright (C) 2013 Paulo Farinatti et al. This is an open access article distributed under the Creative Commons Attribution License, which permits unrestricted use, distribution, and reproduction in any medium, provided the original work is properly cited.

\begin{abstract}
Objectives. Resistance training may influence the resting metabolic rate (RMR), which is desirable in weight management programs. However, its impact on excess postexercise oxygen consumption (EPOC) is yet to be defined. The study evaluated the contribution of resistance training variables to EPOC. Design. Studies published until November 2011 were systematically reviewed. Methods. MEDLINE, LILACS, SCIELO, Science Citation Index, Scopus, SPORTDiscus, and CINAHL databases were consulted. The methodological quality of studies was assessed by the PEDro 10-point scale. A total of 155 participants (54\% men) aged between $20 \pm 2$ and $34 \pm 14$ years were observed by 16 studies (quality scores ranged from 5 to 7 ), which were organized according to treatment similarity (number of sets, intensity, rest interval, speed of movement, and exercise order). Results. Training volume seemed to influence both EPOC magnitude and duration, whereas workload influenced mostly the magnitude. Short rest intervals $(<60 \mathrm{~s})$ increased the EPOC magnitude, but not the overall energy expenditure. Conclusion. Resistance training with high intensity and volume, performed with short rest intervals (as in circuit training), probably have greater impact on EPOC. Methodological procedures, particularly time of post-exercise observation and RMR assessment, should be standardized to an appropriate quantification of the actual influence of resistance training on EPOC.
\end{abstract}

\section{Introduction}

In the recovery period after exercise there is an increase in $\mathrm{VO}_{2}$ termed the "excess postexercise oxygen consumption" (EPOC). The EPOC represents the number of calories expended (above resting values) after an exercise bout. The EPOC effect is greatest soon after the exercise is completed and decreases over time. Aerobic training is considered as the most effective exercise to increase energy expenditure (EE) and EPOC [1], but resistive training (RT) has been also shown to have a favorable impact [2-7]. It is accepted that during recovery the EPOC goes along with greater fat burn, which is a desirable effect to weight loss purposes $[3,8]$.

Although some studies have suggested that the contribution of RT to increase EE would be due to the exercise session alone $[8,9]$, others indicated that a higher oxygen uptake $\left(\mathrm{VO}_{2}\right)$ after the end of exercise would also play an important role $[5,10]$. The possible contribution of RT to EPOC has been therefore investigated [10-19]. The available research suggests that RT may elicit a valuable EPOC to weight management-some studies have even found significantly greater EPOC response after RT compared to aerobic exercise bouts $[20,21]$.

Nevertheless a number of questions remain unclear. Variation in study design (exercises performed, number of repetitions, workload, recovery intervals, and energy assessment protocol) and sample (age, sex, body composition, training level, and diet) may influence the EPOC. Hence large variations in the EPOC following RT have been reported $[3,8-12,15-30]$. 
In brief, many gaps persist concerning the relative influence of RT variables on EPOC. Thus, this study aimed to investigate, by means of systematic review, the influence of some RT variables on the EPOC magnitude and duration: number of sets, intensity (workload), rest intervals, training mode, speed of movement, and exercise order.

\section{Methods}

2.1. Inclusion Criteria. The following inclusion criteria were adopted to select the studies: (a) experimental studies aiming exclusively to compare the influence of RT on metabolic responses, including EPOC; (b) target population composed by healthy individuals; (c) RT performed with isotonic devices. Studies published in English, Spanish, and Portuguese were found through electronic searching.

2.2. Searching for Primary Studies. Titles and abstracts were reviewed independently by two reviewers to identify those meeting the inclusion criteria. The search was systematically conducted, without date limits in Medline, EMBASE, Lilacs, Scielo, Science Citation Index, National Library of Medicine, Scopus, SPORTDiscus, and CINAHL databases. In Medline, a translation of the optimum OVID search strategy [31] was combined with specific search terms for each topic.

The following steps were used in the prospection of studies: (a) search algorithms with recognition of specialized literature, (b) reference lists of the found studies, and (c) manual search of articles in journals not virtually found. The following keywords were used, resistance exercise and postexercise oxygen consumption or postexercise energy expenditure or energy expenditure or resting metabolic rate or metabolic responses or EPOC. The same possibilities were tested using other terms usually related to RT, such as weight training, weight lifting, and strength training. If a study did not present data necessary for analysis, additional information was requested from the authors. The last search was performed in November 10, 2011.

2.3. Assessment of Study Quality and Data Synthesis. The quality of included studies was independently assessed by two researchers, and disagreements were decided by a third researcher. The researchers were asked to evaluate the studies with regard to the control of potential confounding factor-sample selection, statistical treatment, strength testing including its reproducibility, resting metabolic rate (RMR) assessment including its reproducibility, and diet control. If the researchers considered that a given study neglected two or more of these factors, it was excluded from the review. Methodological quality was assessed by an adaptation of the PEDro scale, which is based on the Delphi list [32]. Some criteria were adapted in order to be coherent with the reviewed studies, since most of them were not case control or typical experimental studies. The studies were grouped by treatment similarity (number of sets, workload, rest intervals, training mode, speed of movement, and exercise order), and each variable was analyzed separately. Data expressed in kilojoules were converted into calories to allow comparison between studies.

\section{Results}

3.1. Trial Characteristics. Primary search of all sources retrieved a total of 1507 citations, 40 of which were judged to merit scrutiny of the full article and 16 of which met the inclusion criteria $[11,12,15-19,22-28,30]$. Table 1 presents a summary of the studies eligible for this systematic review.

The selected studies included men and women in a close proportion ( $50.4 \%$ versus $49.6 \%$ of the participants, resp.), with age from 21 to 34 yrs. Training intensities were reported in terms of percent age of one repetition maximum (\%RM) $[11,17-19,22-24,28,30]$, number of RM [12, 15, 16, 26, 27], and rate of perceived exertion [17]. The exercises included more often were the bench press [11, 12, 16-19, 23, 25, 27, 28], leg extension $[11,12,17,23,25-28]$, biceps curl $[11,12$, $18,23,25-27]$, and leg press, [17, 27, 28], among other less common ones (chest fly, seated row, military press, triceps extension, lat pull down, squat machine, and shoulder press) $[11,12,15-19,22-28,30]$. The number of sets ranged from one $[15,19]$ to six $[11]$ and the rest intervals ranged from $20 \mathrm{~s}$ [12] to $5 \mathrm{~min}$ [22]. The intensity ranged from $25 \%$ [23] to $90 \%$ [19] of $1 \mathrm{RM}$, while the speed of movement ranged from $1 \mathrm{~s}$ to $10 \mathrm{~s}$ in concentric and eccentric phases [23].

The EPOC magnitude was expressed either as kcal [11, $12,15-18,22,23,25,28,30]$ or KJ $[15,19,25,28]$. Reported EE ranged from $4.1 \mathrm{kcal}$ [19] to $114 \mathrm{kcal}$ [11]. The EPOC duration was as short as $5 \mathrm{~min}$ [27] and as long as $900 \mathrm{~min}$ [11].

3.2. Methodological Quality of Included Studies. The betweenresearcher reliability of quality ratings was acceptable $($ kappa $=0.77$, agreement $=89 \%)$. The methodological quality of the studies was moderate to high (Table 2). The range of quality scores was 6-7 out of 10 . Not all criteria on the PEDro scale could be satisfied (e.g., blinding of evaluators is difficult). If a study did not clearly specify that a criterion was met, it was scored as not satisfying the criterion.

3.3. Influence of Training Variables on the EPOC. Only three studies investigated the impact of the number of sets on the EPOC $[11,15,24]$. Melby et al. [11] combined two training methods (single set versus circuit); Haddock and Wilkin [15] manipulated both number of sets and rest intervals in order to keep the training volume constant. The work by Mazzetti et al. [24] modified the number of sets, but also the workload and speed of movement-therefore it cannot be considered as specifically addressing this issue. Altogether the results of these studies indicated that the number of sets per se would not influence the EPOC.

The influence of rest intervals on muscle fatigue has been investigated [33], but the possible effect of this variable on the EPOC has not been given the same attention. We found only three studies investigating the specific influence of rest intervals between sets and exercises on EPOC magnitude and duration $[12,22,34]$. In general, the magnitude increased 


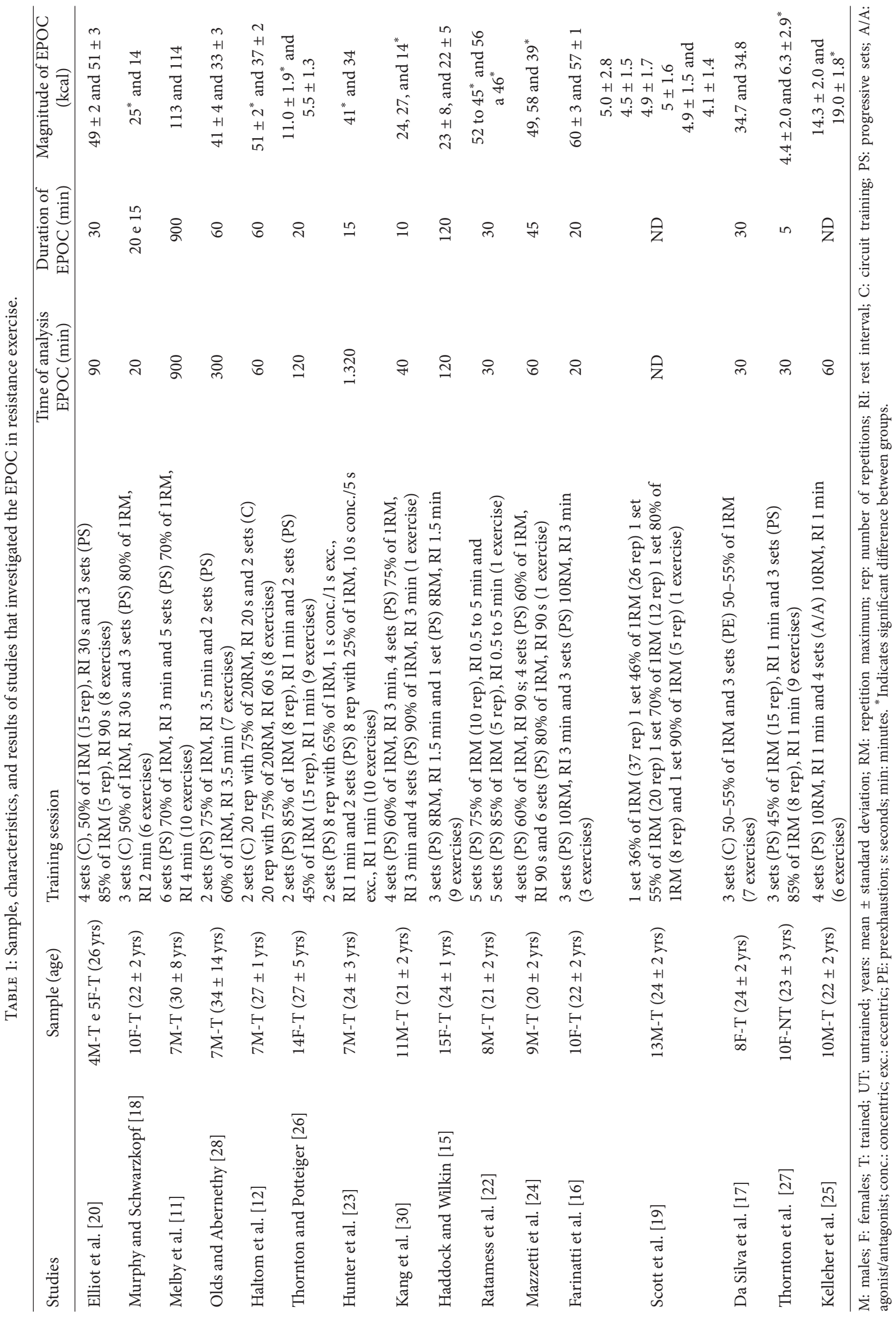




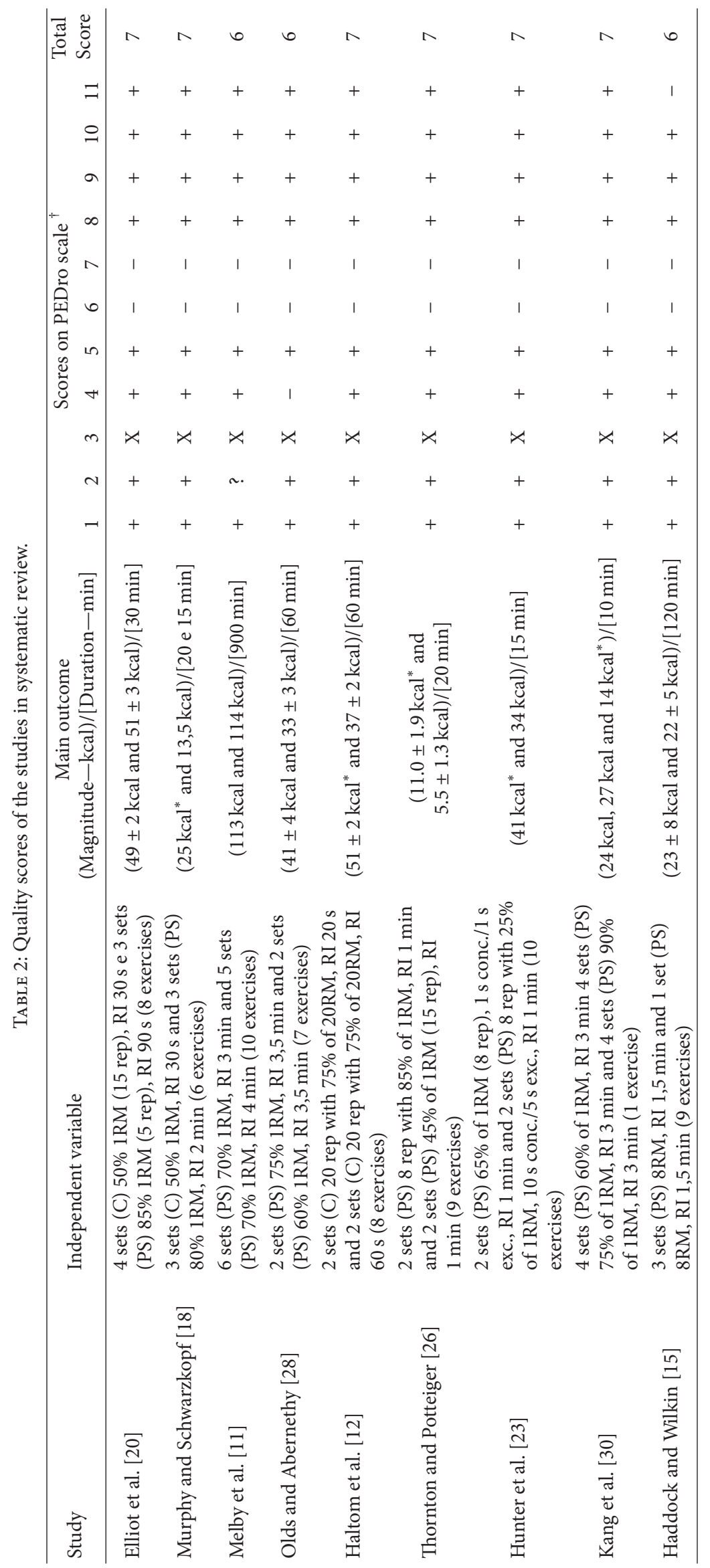




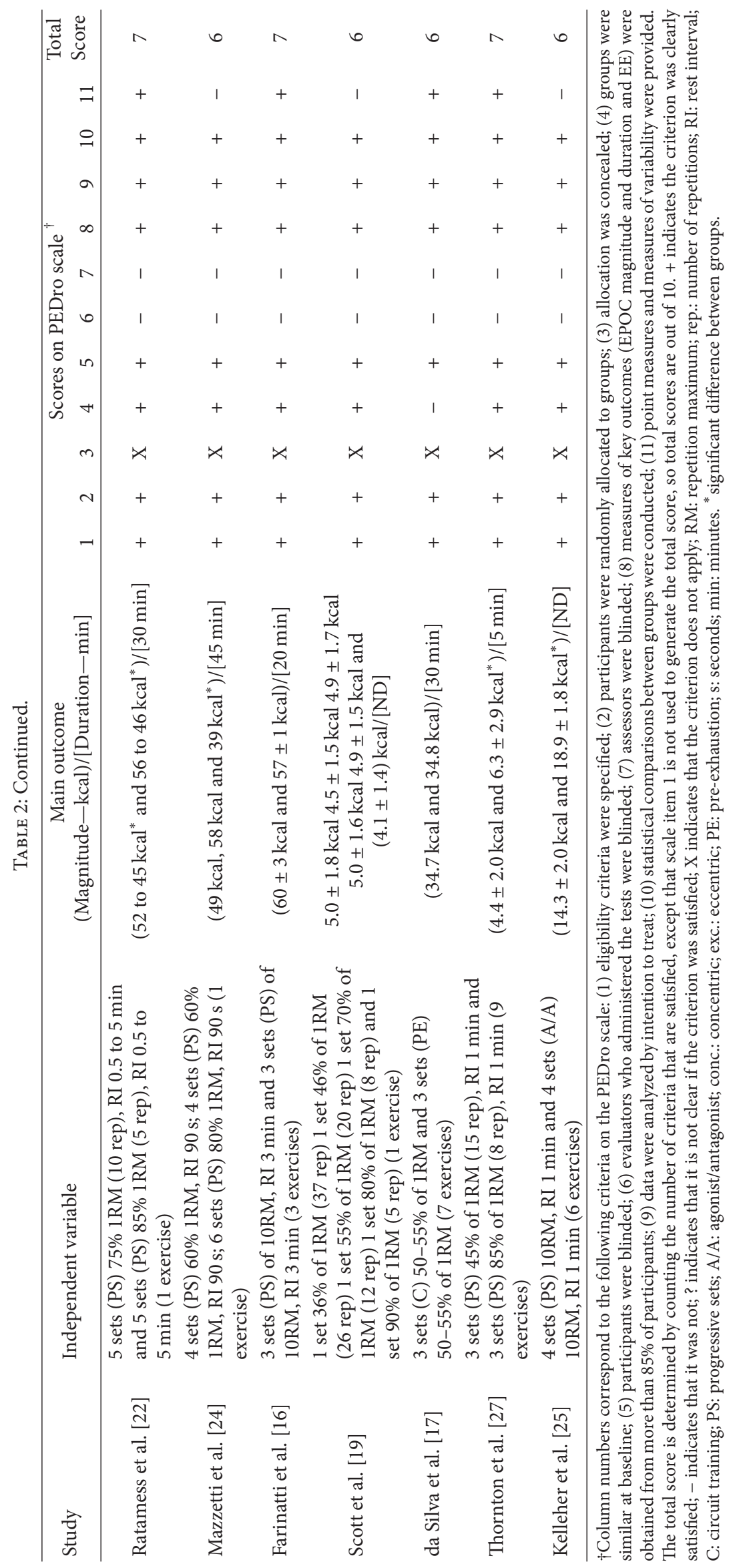


when the rest intervals were shorter, whereas the $\mathrm{VO}_{2}$ within the exercise sessions decreased.

The training intensity is the most investigated training variable with regard to EE and is usually expressed either in terms of percentage of $1 \mathrm{RM}$ or maximal repetitions. A total of nine studies on the relationship between EPOC and RT intensity were included in the present review $[18,19,22-24$, $26-28,30]$. The accumulated evidence suggests that higher load increases the EPOC magnitude, but not its duration. The variation in the speed of movement is frequently applied to change RT intensity [35]. Nonetheless very few studies investigated the effects of this variable on the EPOC. We found two studies $[23,24]$ comparing the EPOC following RT performed with different velocities. The results are not consistent, but it is possible that slower contractions induce higher EPOC magnitude in comparison with explosive contractions, at least in sessions matched for intensity and volume.

The possible effect of exercise ordering on the EPOC has not been extensively studied. Only three studies [16, 17, 25] investigated this issue. Considering that training methods can be in some extent related to the exercise order, other three studies were analyzed $[12,18]$. The available results are not suggestive that exercise order influences the EPOC. However, circuit sessions may increase its magnitude compared to traditional consecutive sets.

\section{Discussion}

This systematic review evaluated results from 16 studies of moderate to high quality. The results may have been biased by including only studies reported in English, Spanish, and Portuguese. However, when a less sensitive search for studies in other languages was performed, the additional studies that were found did not satisfy the inclusion criteria. The PEDro scale used to discriminate between studies of different quality is yet to be fully validated, but has been applied in systematic reviews [36], and is unlikely to have produced bias.

4.1. Effect of Number of Sets. Only two studies addressed the influence of the number of sets on EPOC. Haddock and Wilkin [15] tested whether sessions with similar intensity and different volumes increased the EPOC in trained women. Participants underwent two protocols consisting of either one or three (circuit) sets of nine exercises performed with $8 \mathrm{RM}$ to volitional fatigue. The EPOC was assessed during $120 \mathrm{~min}$. The $\mathrm{VO}_{2}$ during the exercises was greater in the 3-set over 1 -set protocol, but the EE along 120 min recovery was not significantly different $(22.3 \mathrm{kcal}$ versus $22.5 \mathrm{kcal}$, resp.). These results suggest that the EPOC would be influenced by training volume, at least within protocols performed with similar load.

The study by Melby et al. [11] ratified the idea that the number of sets would not influence the EPOC, at least when it is the only independent variable. In a first experiment, seven men performed 90 min weight-lifting including six sets of 10 exercises with $70 \% 1 \mathrm{RM}$, and 3 min rest intervals. In a second experiment they underwent a protocol with similar duration including five sets of the same exercises and workload, but $4 \mathrm{~min}$ rest intervals. Postexercise metabolic rate was measured for $2 \mathrm{~h}$ and compared with preexercise baseline. The RMR was assessed $15 \mathrm{~h}$ after completion of the workout. The EPOC remained similarly elevated after both protocols (approximately 10\%) during the $2 \mathrm{~h}$ recovery period. However, the RMR measured after $15 \mathrm{~h}$ was $9.4 \%$ higher after the first protocol and $4.7 \%$ higher after the second protocol, in comparison with baseline. These results suggest that the modification of the number of sets and rest intervals, without significant change in the overall training volume, would not affect the EPOC. On the other hand, the RMR may be influenced and this certainly warrants additional research.

4.2. Effect of Rest Intervals. Haltom et al. [12] investigated the effects of rest intervals (20s versus $60 \mathrm{~s}$ ) upon the magnitude of $1 \mathrm{~h} \mathrm{EPOC} \mathrm{following} \mathrm{two} \mathrm{circuits} \mathrm{of} \mathrm{eight} \mathrm{upper}$ and lower body exercises (20 repetitions at 75\% 20RM). Total EE (exercise + recovery) was somewhat greater in the $60 \mathrm{~s}(277.2 \mathrm{kcal})$ compared to the $20 \mathrm{~s}$ rest interval protocol $(242.2 \mathrm{kcal})$. However, the magnitude of the EPOC was significantly higher in the $20 \mathrm{~s}$ than in the $60 \mathrm{~s}$ protocol $(10.3 \mathrm{~L}$ versus $7.4 \mathrm{~L}$ corresponding to $51.5 \mathrm{kcal}$ versus $37.0 \mathrm{kcal}$, resp.). In other words, shortening the rest intervals induced greater EPOC. In which concerns the EPOC duration, the $\mathrm{VO}_{2}$ did not return to baseline resting values in any of those protocols, which makes difficult to evaluate the EPOC duration.

Ratamess et al. [22] compared the effect of different rest intervals ( $30 \mathrm{~s}, 1 \mathrm{~min}, 2 \mathrm{~min}, 3 \mathrm{~min}$, and $5 \mathrm{~min}$ ) on $\mathrm{EE}$ assessed during $30 \mathrm{~min}$ after the bench press performed with $75 \%$ and $85 \% 1 \mathrm{RM}$. The combination of $85 \% 1 \mathrm{RM}$ and $30 \mathrm{~s}$ interval elicited the higher EPOC magnitude. In the exercise performed with 75\% 1RM the greater EPOC was also observed in the $30 \mathrm{~s}$ compared to the other intervals. Unfortunately the EPOC assessment was again interrupted before the $\mathrm{VO}_{2}$ normalization, precluding inferences about its duration.

More recently, our group [34] observed the effect of different rest intervals ( 1 min versus $3 \mathrm{~min}$ ) on the EPOC measured during $90 \mathrm{~min}$ after protocols with multiple sets and exercises engaging large and small muscle mass (leg press versus chest fly). The duration of the EPOC was about the same after both leg press protocols (about $40 \mathrm{~min}$ ), whereas after the chest fly it was longer when the rest interval was shorter ( $40 \mathrm{~min}$ versus $20 \mathrm{~min}$ ). The total $\mathrm{VO}_{2}$ was not influenced by the rest intervals regardless of the exercise. However, shortening the intervals increased the $\mathrm{VO}_{2}$ in the initial few minutes of recovery, which concurs with the results by Haltom et al. [12] and Ratamess et al. [22]. In a practical perspective, it seems that the shorter the rest intervals, the higher the need to assess the $\mathrm{VO}_{2}$ during recovery to correctly estimate the EE associated with RT.

4.3. Effect of Exercise Intensity. Accumulated evidence indicates that exercise intensity influences the EPOC. Hunter et al. [23] applied two protocols with 2 sets of 8 repetitions of 10 exercises $(65 \% 1 \mathrm{RM}, 1 \mathrm{~s}$ for both concentric and eccentric phases versus 25\% 1RM, $10 \mathrm{~s}$ for the concentric and $5 \mathrm{~s}$ for the eccentric phase). The magnitude of the EPOC was higher for the protocol with greater intensity $(41.0 \mathrm{kcal}$ 
versus $33.5 \mathrm{kcal})$, but the duration was comparable (15 $\mathrm{min})$. Another group [26, 27] observed similar results, detecting greater but not longer $(20 \mathrm{~min})$ EPOC following protocols with $85 \%(11.0 \mathrm{kcal})$ and $45 \% 1 \mathrm{RM}(5.5 \mathrm{kcal})$ (9 exercises, 2 or 3 sets of 8 repetitions).

Some studies failed to demonstrate a relationship between workload and EPOC, but methodological limitations may have affected their results. Murphy and Schwarzkopf [18] observed the EPOC after 6 exercises performed with different intensities (3 successive sets with $80 \% 1 \mathrm{RM}$ and 2 min rest intervals versus 3 circuit sets with $50 \%$ $1 \mathrm{RM}$ and $30 \mathrm{~s}$ rest intervals). The magnitude of the EPOC was greater after the circuit session performed with lower intensity compared to the successive sets session $\left(4.9 \mathrm{~L} \mathrm{O}_{2}\right.$ versus $2.7 \mathrm{~L} \mathrm{O}_{2}$, resp.), but the duration was similar (about $20 \mathrm{~min}$ ). It is worthy to notice that the time to perform the exercises was longer in the protocol with successive sets. Therefore, the EPOC magnitude could be affected by shorter rest intervals between sets, despite the exercise intensity.

Ratamess et al. [22] have not found differences for both magnitude and duration $(30 \mathrm{~min})$ of the EPOC following RT performed with $85 \%(55.9 \mathrm{kcal})$ and $75 \% 1 \mathrm{RM}(51.8 \mathrm{kcal})(5$ sets of bench press and $30 \mathrm{~s}$ intervals). However, the total volume of the sessions was quite low. Moreover, it is probable that the small difference between workloads was not enough to influence the post-exercise $\mathrm{VO}_{2}$. The same problem may have occurred in another study [28] comparing RT with 75\% and $60 \% 1 \mathrm{RM}$ ( 2 sets of 7 exercises, $120 \mathrm{~s}$ rest intervals). Significant differences have neither been found for the EPOC magnitude (41 kcal versus $32 \mathrm{Kcal}$ ) nor duration (60 min).

A single study suggested that the workload could influence the EPOC magnitude and duration [30]. Eleven trained young men underwent 4 sets of squat on sessions differing in intensity and volume (15 repetitions at 60\% $1 \mathrm{RM} ; 10$ repetitions at $75 \% 1 \mathrm{RM}$ and 4 repetitions at 90\% 1RM). Interestingly, the greater EPOC was found after the session performed with $75 \% 1 \mathrm{RM}(27 \mathrm{kcal})$, followed by $60 \% 1 \mathrm{RM}$ $(24 \mathrm{kcal})$, and $90 \% 1 \mathrm{RM}(14 \mathrm{kcal})$. The exercise performed with lower intensities extended the EPOC duration (10 min at $90 \% 1 \mathrm{RM}$ versus $20 \mathrm{~min}$ at $60 \%$ and $75 \% 1 \mathrm{RM}$ ). Unfortunately the protocols were not matched for the work volume; the number of repetitions was considerably lower at 90\% 1RM compared to the other intensities. Therefore it was not possible to determine the relative contribution of intensity and repetitions to the EPOC.

Scott et al. [19] compared protocols with six different intensities and did not observe differences in the EPOC magnitude $(36 \% 1 \mathrm{RM}=5.0 \pm 1.8 \mathrm{kcal}, 46 \% 1 \mathrm{RM}=4.5 \pm$ $1.5 \mathrm{kcal}, 55 \% 1 \mathrm{RM}=4.9 \pm 1.7 \mathrm{kcal}, 70 \% 1 \mathrm{RM}=5.0 \pm 1.6 \mathrm{kcal}$, $80 \% 1 \mathrm{RM}=4.9 \pm 1.5 \mathrm{kcal}, 90 \% 1 \mathrm{RM}=4.1 \pm 1.4 \mathrm{kcal})$. Again the training volume was a main confounding factor and the higher EE was found when a greater volume of work was performed regardless of the post-exercise $\mathrm{VO}_{2}$. No specific information about the EPOC duration was provided, but its magnitude appeared to be linearly related to RT duration and exponentially related to the intensity.

4.4. Effect of Speed of Movement. Results on the influence of the speed of movement on the EPOC are controversial.
Hunter et al. [23] compared the metabolic response to exercises performed with 2 sets of 8 repetitions at usual (or traditional) velocity $(65 \% 1 \mathrm{RM}, 1 \mathrm{~s}$ for both concentric and eccentric phases) and super-slow one (25\% 1RM, $10 \mathrm{~s}$ for the concentric and $5 \mathrm{~s}$ for the eccentric phase). The EPOC magnitude was greater after the traditional compared to the super-slow protocol ( $41 \mathrm{kcal}$ versus $33.5 \mathrm{kcal}$ ) but not the EPOC duration ( $15 \mathrm{~min}$ ). However, these findings could be influenced by the exercise intensity, since the super-slow protocol had lower workload.

Mazzetti et al. [24] compared explosive versus slow contractions on EE during and after three squat exercise protocols. The slow protocol used $2 \mathrm{~s}$ concentric contractions, while the explosive protocol applied $1 \mathrm{~s}$ concentric contractions (8 repetitions, 4 sets, $90 \mathrm{~s}$ rest intervals, $60 \%$ $1 \mathrm{RM})$. The EPOC duration was approximately $45 \mathrm{~min}$ in both protocols, but the magnitude was greater after the explosive protocol $(58.4 \mathrm{kcal})$ compared to slow one $(49.2 \mathrm{kcal})$. This study is interesting because squat protocols were performed with identical repetitions, sets, loads, eccentric speeds, and intervals. The rate of EE was increased by approximately $11 \%$ during the explosive and 5\% after the slow protocol.

In contrast, Dolezal et al. [13] suggested that slower contractions would increase the EPOC due to greater muscle damage. Leg press emphasizing the eccentric movement $(4 \mathrm{~s})$ was performed by trained and untrained participants ( 8 sets with $6 \mathrm{RM})$. The RMR was significantly elevated compared to baseline during $48 \mathrm{~h}$, especially in untrained participants. Unfortunately, this study applied only contractions emphasizing the eccentric phase, with no comparison between different speeds of contraction whatsoever.

4.5. Effect of Exercise Order and Training Methods. We found only one study specifically addressing the effect of exercise ordering on the EPOC. Farinatti et al. [16] investigated the influence of mirrored sequences in three sets to volitional fatigue of upper body exercises performed at 10RM (bench press, shoulder press, and triceps extension) on the work volume and $\mathrm{EE}$. The $\mathrm{VO}_{2}$ was measured within the exercises and $20 \mathrm{~min}$ after the sequences. No difference between sequences was found for net $\mathrm{VO}_{2}$ or 20 min EPOC. However, the arbitrary interruption of $\mathrm{VO}_{2}$ assessment after $20 \mathrm{~min}$ recovery is an important limitation.

Kelleher et al. [25] compared the energy cost of reciprocal supersets (alternating multiple sets of high-intensity exercises performed by agonist-antagonist muscle groups with short recovery) and traditional sequential sets (4 sets at 10RM, $1 \mathrm{~min}$ rest intervals). Six exercises for upper and lower body were applied. The $60 \mathrm{~min}$ EPOC was greater after the reciprocal supersets $(19 \mathrm{kcal})$ over traditional sets $(14 \mathrm{kcal})$, despite the similar duration of the protocols $(30 \mathrm{~min}$ and $36 \mathrm{~min}$, resp.). No information was provided on the EPOC duration.

da Silva et al. [17] compared the EE and EPOC after 7 exercises performed with circuit and preexhaustion systems (3 sets of 12 repetitions at $50-55 \% 1 \mathrm{RM}$, no rest intervals, $2 \mathrm{~s}$ in concentric and eccentric phases). No difference was found for the EPOC magnitude within $30 \mathrm{~min}$ recovery (circuit: $34.7 \mathrm{kcal}$ versus pre-exhaustion: $34.8 \mathrm{kcal}$ ), but the 
duration was not analyzed. Several limitations in this study make the interpretation of the results difficult. Firstly, there is no mention to the total work or time to perform each sequence. The fact that rest intervals have been suppressed may have masked possible EPOC differences between protocols. Additionally it is difficult to recognize how untrained individuals could perform without intervals 21 consecutive sets of different exercises without compromising the work volume, even though the workload of 50-55\% 1RM may be considered as moderate. Finally the strategy to fit the mask on the subject during the last set of a given station, in order to allow the $\mathrm{VO}_{2}$ values to be stable before the postexercise assessment, may have biased the results since the used apparatus frequently requires longer time to stabilization, particularly in intermittent exercises [37].

Comparisons after consecutive and circuit sets have been recurrent and, in some way, this can be related to the exercise ordering. Three studies investigated the influence of circuit and traditional exercise order on the EPOC. Murphy and Schwarzkopf [18] applied similar protocols with 6 exercises organized in consecutive and circuit sets (circuit: 3 sets, $50 \% 1 \mathrm{RM}, 30$ s rest intervals versus traditional: 3 sets, $80 \%$ $1 \mathrm{RM}, 60 \mathrm{~s}$ rest intervals), observing higher EPOC after circuit ( $25 \mathrm{kcal})$ over traditional training ( $13.5 \mathrm{kcal})$. Similar findings were reported by Haltom et al. [12] - circuit (51 kcal) versus consecutive sets $(37 \mathrm{kcal})$ (2 sets of 20 repetitions of 8 exercises at $75 \%$ 20RM). The circuit session was significantly shorter than the consecutive sets. To control bias due to differences in training duration, Elliot et al. [20] compared the EPOC following 8 exercises organized in consecutive and circuit sessions matched for the duration $(40 \mathrm{~min}$ ) (circuit: 4 sets of 15 repetitions at $50 \% 1 \mathrm{RM}, 30 \mathrm{~s}$ rest intervals versus consecutive: 3 sets of 5 repetitions at 85\% 1RM, 1 min intervals). The EPOC magnitude was similar across the sequences (circuit: $49 \mathrm{kcal}$ versus consecutive: $51 \mathrm{kcal}$ ). Again no information was given about EPOC duration.

It is worthy to notice that in most studies circuit sessions had shorter rest intervals. So, it is feasible that the influence of the training method on EPOC was influenced by greater accumulated fatigue [33]. Actually the relationship between exercise order, rest intervals, and work volume adds significant bias on post-exercise $\mathrm{VO}_{2}$, which warrants future research on the particular role of each variable on EPOC magnitude and duration.

4.6. Methodological Issues in EPOC Analysis. Several mechanisms have been suggested to explain the EPOC. Factors as body temperature, hormonal variables, restoration of muscle energy phosphate stores, and replenishment of $\mathrm{O}_{2}$ in blood and muscles; redistribution of compartmental ions, or lactate removal have been mentioned as possible causes of EPOC rapid and slow phases $[8,13,38-42]$.

Unfortunately, investigation about the specific influence of RT variables on the EPOC curve has been neglected. There are very few studies addressing this issue and variation in study design (exercises performed, number of repetitions, workload, recovery intervals, and energy assessment protocol) which make the comparison difficult between findings. For instance, the EPOC assessment time ranges from as short as $5 \mathrm{~min}[19,43]$ to as long as $60 \mathrm{~min}$ [12] or $24 \mathrm{~h} \mathrm{[14].} \mathrm{Some}$ studies adopted fixed periods to assess the EPOC $[12,15-$ $17,22-28,30]$, whereas others considered the return of $\mathrm{VO}_{2}$ to preexercise or RMR values as endpoints $[11,18,19]$. Additionally many studies discarded the first minutes of the EPOC, therefore overlooking its rapid phase even though approximately $1 / 3$ of the total $\mathrm{VO}_{2}$ recovery takes place during this period [39]. In the present paper, only five studies [16, $22,26,27,34]$ assessed the EPOC immediately after the end of RT.

The influence of rest intervals should also be considered. It has been previously shown that measuring the $\mathrm{VO}_{2}$ during each set may underestimate the peak and mean response, especially if short rest intervals are used. However, if the intervals are too long, the absolute $\mathrm{VO}_{2}$ within a given session may be artificially high. It is therefore important to standardize the rest intervals between sets and exercises to prevent assessment bias: short intervals result in high EPOC, but less EE within the exercise session. On the other hand, longer intervals result in lower EPOC, but greater EE during the session $[16,34]$.

In a few words, the magnitude and duration of the EPOC within RT studies may have been in great extent influenced by the adopted assessment criteria. Not surprisingly, the total $\mathrm{VO}_{2}$ reported by the available research falls within a wide range, even when the sessions are of comparable volume $[9,12,22,23,44]$. This is certainly a central aspect that warrants future research.

\section{Conclusion}

The comparison between studies investigating the influence of RT variables on EPOC is made difficult by the variety of methodological approaches and lack of standardization of procedures, particularly the assessment of $\mathrm{VO}_{2}$ during the post-exercise recovery. However, the present paper indicated that the following. (a) changing the number of sets without variation in training volume should not affect the EPOC; (b) shorter rest intervals between sets and exercises $(<60 \mathrm{~s})$ increase the EPOC magnitude, but not the overall EE; (c) the exercise intensity seems to influence the EPOC magnitude, but not its duration; (d) there is no solid evidence indicating that the speed of movement or exercise order influence the EPOC; (e) Considering a given work volume, the EPOC magnitude appears to be greater after circuit training compared to consecutive traditional sessions.

In brief, the accumulated evidence suggests that RT volume would be more important than intensity alone to optimize the EPOC. In this sense, circuit training with short rest intervals would be the best strategy to increase the EPOC after RT. Future research is warranted to confirm these hypotheses within actual RT sets.

The included studies indicate that RT may help increasing EPOC and EE, even though some of the differences in the EPOC between resistance exercises with different methodology may seem very small from a practical perspective. However, any additional caloric expenditure following exercise may contribute to long-term weight management. It must be remembered that weight-control benefits of EPOC 
should happen over a significant time period. Thus, even acknowledging that the EPOC induced by a single exercise session would not represent a great impact on overall EE, the cumulative effect of sequential RT sessions may be relevant in the context of long-term programs.

\section{Acknowledgments}

This study was partially supported by Grants from the Brazilian Council for the Technological and Research Development $(\mathrm{CNPq})$ and Carlos Chagas Foundation for the Research Support in the Rio de Janeiro State (FAPERJ). The authors have no conflict of interests that are directly relevant to the content of this paper.

\section{References}

[1] J. E. Donnelly, S. N. Blair, J. M. Jakicic, M. M. Manore, J. W. Rankin, and B. K. Smith, "Appropriate physical activity intervention strategies for weight loss and prevention of weight regain for adults," Medicine and Science in Sports and Exercise, vol. 41, no. 2, pp. 459-471, 2009.

[2] K. L. Osterberg and C. L. Melby, "Effect of acute resistance exercise on postexercise oxygen consumption and resting metabolic rate in young women," International Journal of Sport Nutrition, vol. 10, no. 1, pp. 71-81, 2000.

[3] M. J. Ormsbee, J. P. Thyfault, E. A. Johnson, R. M. Kraus, D. C. Myung, and R. C. Hickner, "Fat metabolism and acute resistance exercise in trained men," Journal of Applied Physiology, vol. 102, no. 5, pp. 1767-1772, 2007.

[4] E. P. Kirk, J. E. Donnelly, B. K. Smith et al., "Minimal resistance training improves daily energy expenditure and fat oxidation," Medicine and Science in Sports and Exercise, vol. 41, no. 5, pp. 1122-1129, 2009.

[5] R. W. Bryner, I. H. Ullrich, J. Sauers et al., "Effects of resistance versus aerobic training combined with an 800 calorie liquid diet on lean body mass and resting metabolic rate," Journal of the American College of Nutrition, vol. 18, no. 2, pp. 115-121, 1999.

[6] W. J. Kraemer, J. S. Volek, K. L. Clarck et al., "Physiological adaptations to a weight-loss dietary regimem and exercise programs in women," Journal of Applied Physiology, vol. 83, pp. 270-279, 1997.

[7] E. T. Poehlmam, W. F. Denino, T. Beckett et al., "Effects of endurance and resistance training on total daily energy expenditure in young women: a controlled randomized trial," The Journal of Clinical Endocrinology and Metabolism, vol. 87, pp. 104-109, 2002.

[8] C. A. Binzen, P. D. Swan, and M. M. Manore, "Postexercise oxygen consumption and substrate use after resistance exercise in women," Medicine and Science in Sports and Exercise, vol. 33, no. 6, pp. 932-938, 2001.

[9] W. T. Phillips and J. R. Ziuraitis, "Energy cost of the ACSM single-set RT protocol," The Journal of Strength and Conditioning Research, vol. 17, pp. 350-355, 2003.

[10] M. D. Schuenke, R. P. Mikat, and J. M. McBride, "Effect of an acute period of resistance exercise on excess post-exercise oxygen consumption: implications for body mass management," European Journal of Applied Physiology, vol. 86, no. 5, pp. 411-417, 2002.

[11] C. Melby, C. Scholl, G. Edwards, and R. Bullough, "Effect of acute resistance exercise on postexercise energy expenditure and resting metabolic rate," Journal of Applied Physiology, vol. 75, no. 4, pp. 1847-1853, 1993.

[12] R. W. Haltom, R. R. Kraemer, R. A. Sloan, E. P. Hebert, K. Frank, and J. L. Tryniecki, "Circuit weight training and its effects on excess postexercise oxygen consumption," Medicine and Science in Sports and Exercise, vol. 31, no. 11, pp. 1613-1618, 1999.

[13] B. A. Dolezal, J. A. Potteiger, D. J. Jacobsen, and S. H. Benedict, "Muscle damage and resting metabolic rate after acute resistance exercise with an eccentric overload," Medicine and Science in Sports and Exercise, vol. 32, no. 7, pp. 1202-1207, 2000.

[14] E. L. Melanson, T. A. Sharp, H. M. Seagle et al., "Twenty-fourhour metabolic responses to resistance exercise in women," The Journal of Strength and Conditioning Research, vol. 19, no. 1, pp. 61-66, 2005.

[15] B. L. Haddock and L. D. Wilkin, "Resistance training volume and post exercise energy expenditure," International Journal of Sports Medicine, vol. 27, no. 2, pp. 143-148, 2006.

[16] P. T. V. Farinatti, R. Simão, W. D. Monteiro, and S. J. Fleck, "Influence of exercise order on oxygen uptake during strength training in young women," The Journal of Strength and Conditioning Research, vol. 23, no. 3, pp. 1037-1044, 2009.

[17] R. L. da Silva, M. A. Brentano, and L. F. M. Kruel, "Effects of different strength training methods on postexercise energetic expenditure," The Journal of Strength and Conditioning Research, vol. 24, no. 8, pp. 2255-2260, 2010.

[18] E. Murphy and R. Schwarzkopf, "Effects of standard set and circuit weight training on excess postexercise oxygen consumption," The Journal of Applied Sport Science Research, vol. 6, pp. 88-91, 1992.

[19] C. B. Scott, B. H. Leighton, K. J. Ahearn, and J. J. McManus, "Aerobic, anaerobic, and excess postexercise oxygen consumption energy expenditure of muscular endurance and strength: 1-set of bench press to muscular fatigue," The Journal of Strength and Conditioning Research, vol. 25, no. 4, pp. 903-908, 2011.

[20] D. L. Elliot, L. Goldberg, and K. S. Kuehl, "Effect of resistance training on excess post-exercise oxygen consumption," The Journal of Applied Sport Science Research, vol. 6, pp. 77-81, 1992.

[21] C. A. Gillette, R. C. Bullough, and C. L. Melby, "Postexercise energy expenditure in response to acute aerobic or resistive exercise," International Journal of Sport Nutrition, vol. 4, no. 4, pp. 347-360, 1994.

[22] N. A. Ratamess, M. J. Falvo, G. T. Mangine, J. R. Hoffman, A. D. Faigenbaum, and J. Kang, "The effect of rest interval length on metabolic responses to the bench press exercise," European Journal of Applied Physiology, vol. 100, no. 1, pp. 1-17, 2007.

[23] G. R. Hunter, D. Seelhorst, and S. Snyder, "Comparison of metabolic and heart rate responses to super slow versus traditional RT," The Journal of Strength and Conditioning Research, vol. 17, pp. 76-81, 2003.

[24] S. Mazzetti, M. Douglass, A. Yocum, and M. Harber, "Effect of explosive versus slow contractions and exercise intensity on energy expenditure," Medicine and Science in Sports and Exercise, vol. 39, no. 8, pp. 1291-1301, 2007.

[25] A. R. Kelleher, K. J. Hackney, T. J. Fairchild, S. Keslacy, and L. L. Ploutz-Snyder, "The metabolic costs of reciprocal supersets versus traditional resistance exercise in young recreationally active adults," The Journal of Strength and Conditioning Research, vol. 24, no. 4, pp. 1043-1051, 2010.

[26] M. K. Thornton and J. A. Potteiger, "Effects of resistance exercise bouts of different intensities but equal work on EPOC," Medicine and Science in Sports and Exercise, vol. 34, no. 4, pp. 715-722, 2002. 
[27] M. K. Thornton, S. J. Rossi, and J. L. Mcmillan, "Comparison of two different resistance training intensities on excess postexercise oxygen consumption in african american women who are overweight," The Journal of Strength and Conditioning Research, vol. 25, no. 2, pp. 489-496, 2011.

[28] T. S. Olds and P. J. Abernethy, "Postexercise oxygen consumption following heavy and light resistance exercise," The Journal of Strength and Conditioning Research, vol. 7, pp. 147-152, 1993.

[29] R. A. Robergs, T. Gordon, J. Reynolds, and T. B. Walker, "Energy expenditure during bench press and squat exercises," The Journal of Strength and Conditioning Research, vol. 21, no. 1, pp. 123-130, 2007.

[30] J. Kang, J. R. Hoffman, J. Im et al., "Evaluation of physiological responses during recovery following three resistance exercise programs," The Journal of Strength and Conditioning Research, vol. 19, no. 2, pp. 305-309, 2005.

[31] K. Dickersin, R. Scherer, and C. Lefebvre, "Identifying relevant studies for systematic reviews," British Medical Journal, vol. 309, no. 6964, pp. 1286-1291, 1994.

[32] A. P. Verhagen, H. C. W. de Vet, R. A. de Bie et al., "The Delphi list: a criteria list for quality assessment of randomized clinical trials for conducting systematic reviews developed by Delphi consensus," Journal of Clinical Epidemiology, vol. 51, no. 12, pp. 1235-1241, 1998.

[33] B. F. de Salles, R. Simão, F. Miranda, J. Da Silva Novaes, A. Lemos, and J. M. Willardson, "Rest interval between sets in strength training," Sports Medicine, vol. 39, no. 9, pp. 766-777, 2009.

[34] P. T. V. Farinatti and A. G. Castinheiras Neto, "The effect of between-set rest intervals on the oxygen uptake during and following resistance exercise sessions performed with large and small muscle mass," The Journal of Strength and Conditioning Research, vol. 25, pp. 3181-3190, 2011.

[35] American College of Sports Medicine, ACSM's Guidelines for Exercise Testing and Prescription, Lippincott Williams \& Wilkins, Philadelphia, Pa, USA, 8th edition, 2009.

[36] R. D. Herbert and M. Gabriel, "Effects of stretching before and after exercising on muscle soreness and risk of injury: systematic review," British Medical Journal, vol. 325, no. 7362, pp. 468-470, 2002.

[37] J. Myers, D. Walsh, M. Sullivan, and V. Froelicher, "Effect of sampling on variability and plateau in oxygen uptake," Journal of Applied Physiology, vol. 68, no. 1, pp. 404-410, 1990.

[38] E. Børsheim and R. Bahr, "Effect of exercise intensity, duration and mode on post-exercise oxygen consumption," Sports Medicine, vol. 33, no. 14, pp. 1037-1060, 2003.

[39] J. Bangsbo, P. D. Gollnick, T. E. Graham et al., "Anaerobic energy production and $\mathrm{O} 2$ deficit-debt relationship during exhaustive exercise in humans," Journal of Physiology, vol. 422, pp. 539-559, 1990.

[40] R. Bahr, "Excess postexercise oxygen consumption: magnitude, mechanisms and practical implications," Acta Physiologica Scandinavica, Supplement, vol. 144, no. 605, pp. 1-70, 1992.

[41] C. B. Scott, "Re-interpreting anaerobic metabolism: an argument for the application of both anaerobic glycolysis and excess post-exercise oxygen consumption (EPOC) as independent sources of energy expenditure," European Journal of Applied Physiology and Occupational Physiology, vol. 77, no. 3, pp. 200-205, 1998.

[42] G. A. Gaesser and G. A. Brooks, "Metabolic bases of excess postexercise oxygen consumption: a review," Medicine and Science in Sports and Exercise, vol. 16, no. 1, pp. 29-43, 1984.
[43] C. E. Pichon, G. R. Hunter, M. Morris, R. L. Bond, and J. Metz, "Blood pressure and heart rate response and metabolic cost of circuit versus traditional weight training," The Journal of Strength and Conditioning Research, vol. 10, no. 3, pp. 153-156, 1996.

[44] M. A. Burleson Jr., H. S. O’Bryant, M. H. Stone, M. A. Collins, and T. Triplett-McBride, "Effect of weight training exercise and treadmill exercise on post-exercise oxygen consumption," Medicine and Science in Sports and Exercise, vol. 30, no. 4, pp. 518-522, 1998. 

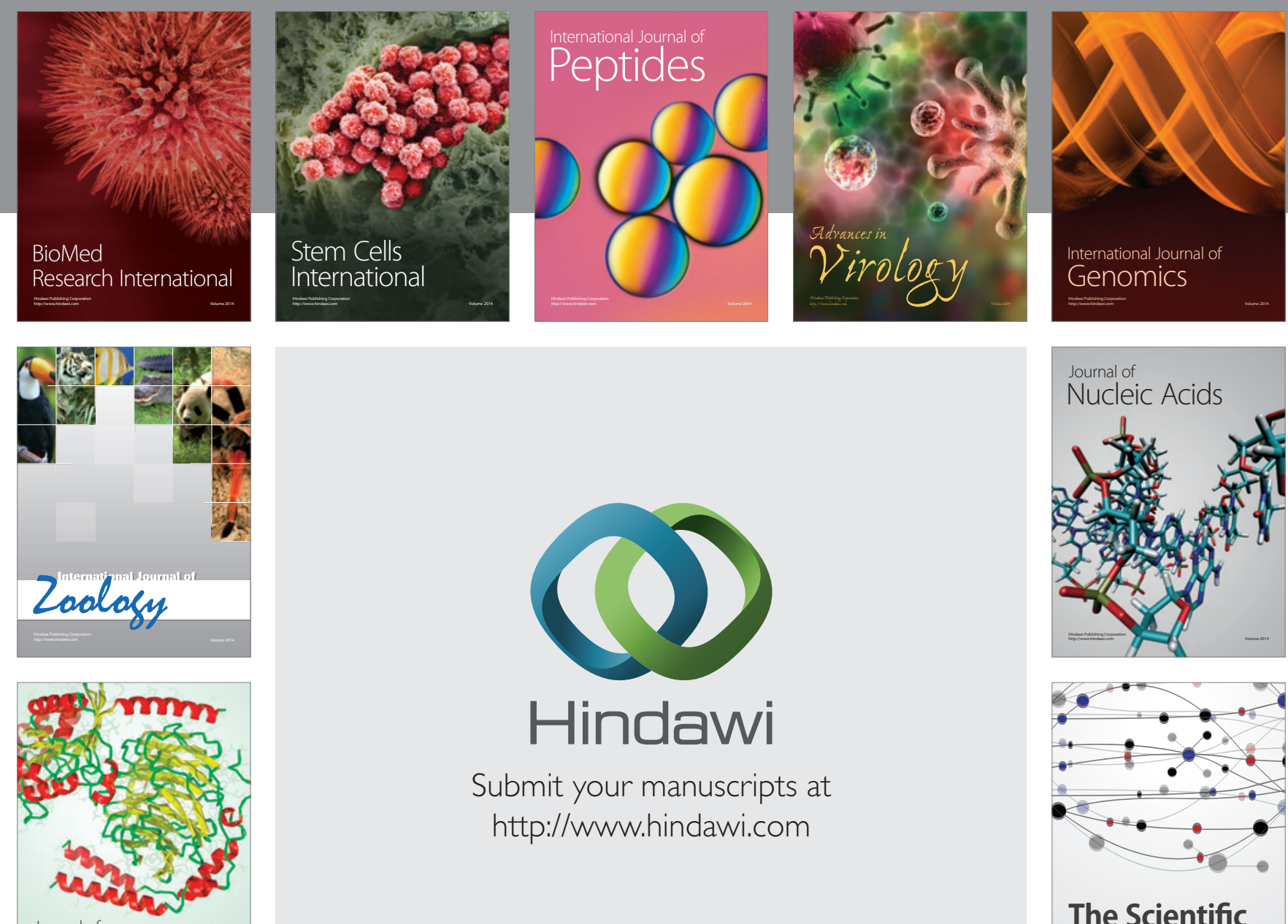

Submit your manuscripts at

http://www.hindawi.com

Journal of
Signal Transduction
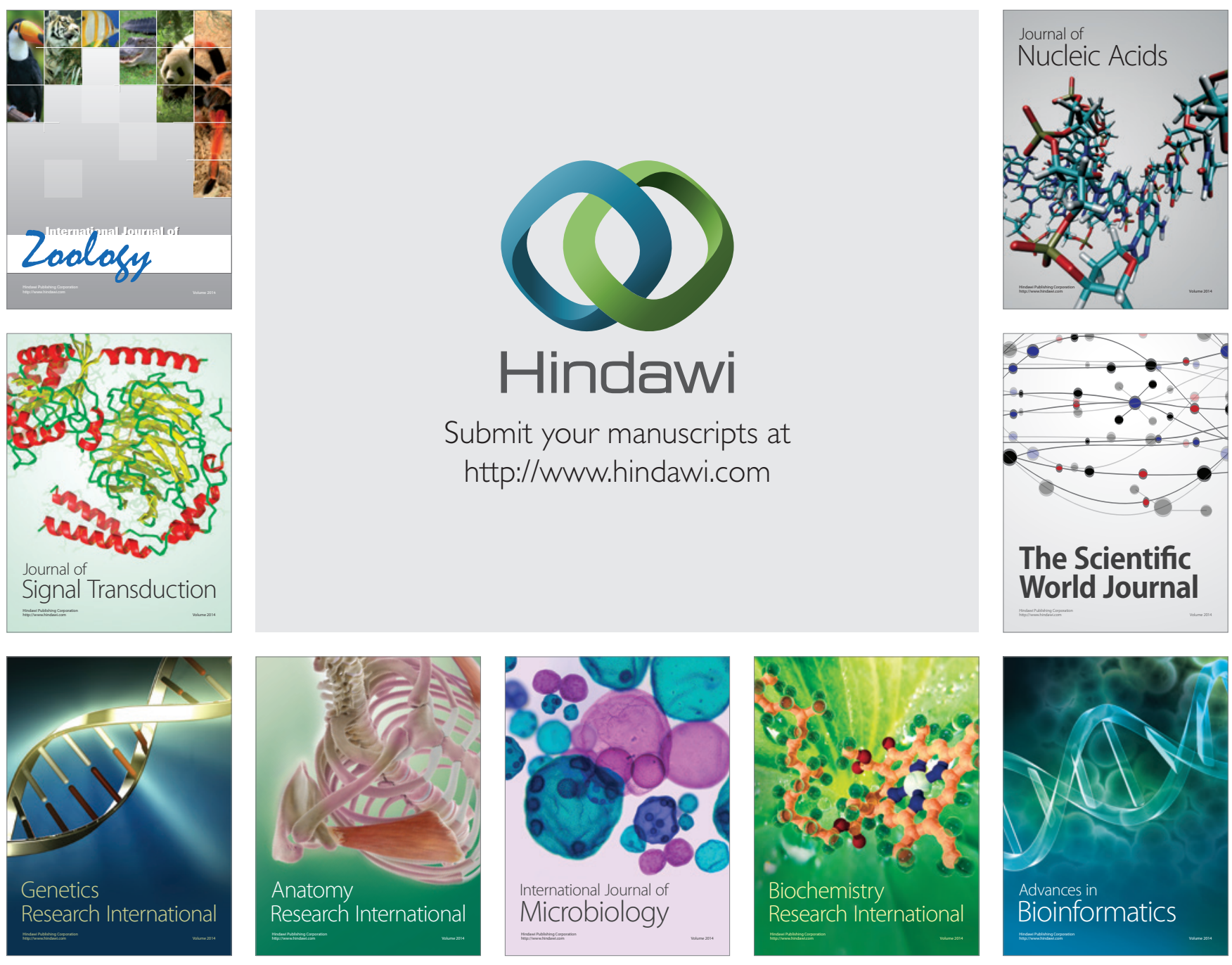

The Scientific World Journal
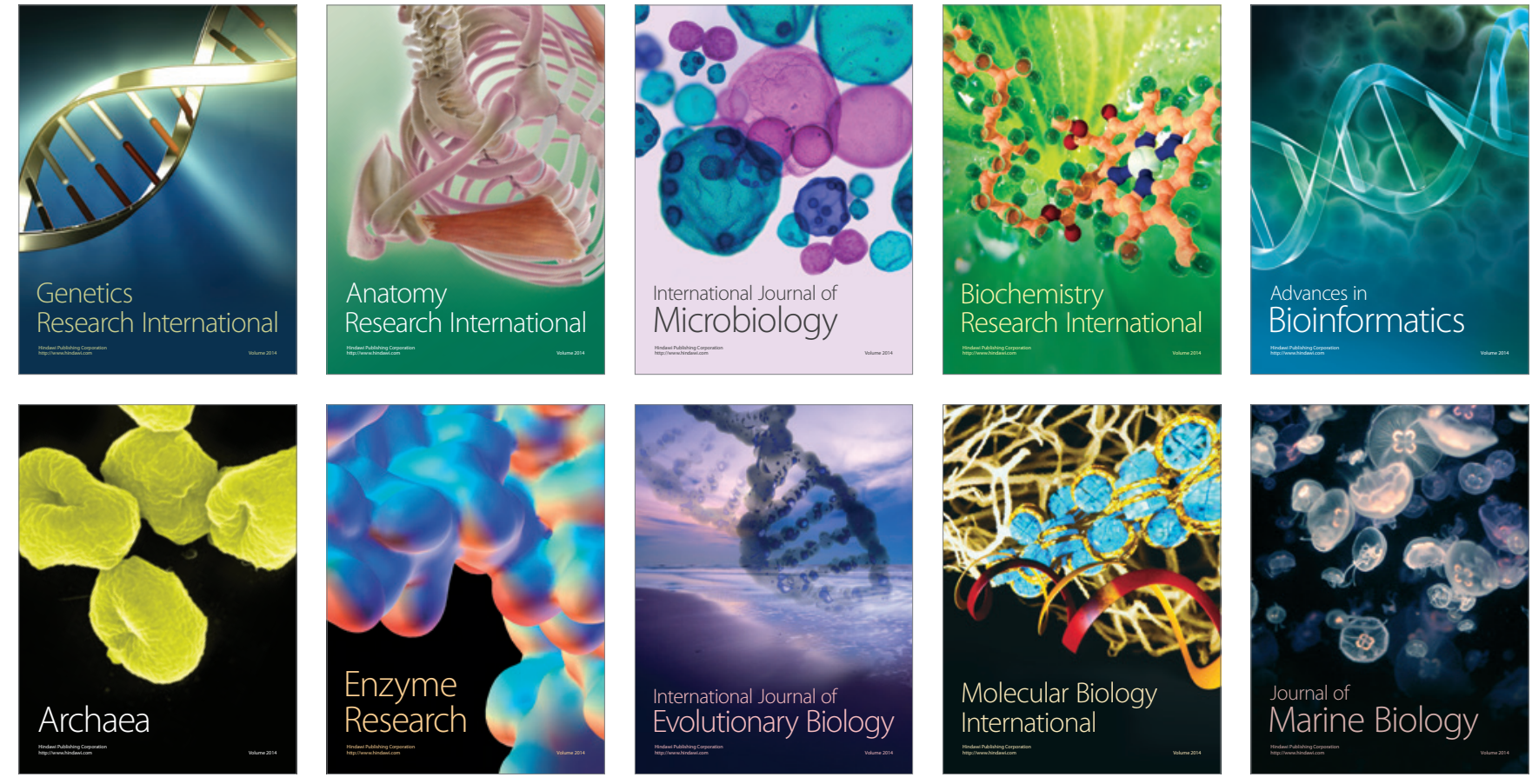